\title{
Massive infection of a song thrush by Mesocestoides sp. (Cestoda) tetrathyridia that genetically match acephalic metacestodes causing lethal peritoneal larval cestodiasis in domesticated mammals
}

\author{
Petr Heneberg ${ }^{1 *} \mathbb{D}$, Boyko B. Georgiev², Jiljí Sitko ${ }^{3}$ and Ivan Literák ${ }^{4}$
}

\begin{abstract}
Background: Peritoneal larval cestodiasis induced by Mesocestoides Vaillant, 1863 (Cyclophyllidea: Mesocestoididae) is a common cause of severe infections in domestic dogs and cats, reported also from other mammals and less frequently from birds. However, there is a limited knowledge on the taxonomy of causative agents of this disease.

Results: In the present study, we investigated a massive, likely lethal, infection of a song thrush Turdus philomelos (Passeriformes: Turdidae) by Mesocestoides sp. tetrathyridia. We performed combined morphological and phylogenetic analysis of the tetrathyridia and compared them with the materials obtained previously from other birds and mammals. The metrical data fitted within the wide range reported by previous authors but confirmed the limited value of morphological data for species identification of tetrathyridia of Mesocestoides spp. The molecular analyses suggested that the isolates represented an unidentified Mesocestoides sp. that was previously repeatedly isolated and sequenced in larval and adult forms from domestic dogs and cats in Europe, the Middle East and North Africa. In contrast to the present study, which found encysted tetrathyridia, four of the five previous studies that identified the same species described infections by acephalic metacestodes only.

Conclusions: The tetrathyridia of the examined Mesocestoides sp. are described in the present study for the first time. However, the possible match with the species that were previously reported to infect birds remains uncertain. The phylogenetic analyses also suggested the rejection of two cases that were previously identified as Mesocestoides corti as they were likely caused by the same species as in the presently reported infection case. The newly provided DNA sequences should allow the assignment to species in the future, when adults of the genus Mesocestoides are more thoroughly sequenced.
\end{abstract}

Keywords: Acephalic metacestodes, Cestoda, Lethal infection, Mesocestoides, Phylogenetic analysis, Tetrathyridia

\section{Background}

Adult cestodes of the genus Mesocestoides Vaillant, 1863 (Cyclophyllidea: Mesocestoididae) are intestinal parasites of carnivore mammals (canids, felids, mustelids or hyaenids) and rarely birds of prey (e.g. [1-4]).

*Correspondence: petr.heneberg@|f3.cuni.cz

${ }^{1}$ Third Faculty of Medicine, Charles University, Prague, Czechia

Full list of author information is available at the end of the article
Mesocestoides spp. have zoonotic potential; records of the infections of non-human primates are frequent [5], but records of infections of humans are sporadic [6, 7]. In addition to the adult individuals, the juvenile stages, commonly known as tetrathyridia and acephalic metacestodes, commonly parasitize a broad range of mammals, and sometimes reptiles, amphibians and birds. The first intermediate host of Mesocestoides spp. is unknown, likely invertebrates $[8,9]$. However, McAllister et al. [10] 
recently suggested that the hypothetical first invertebrate host may be absent and Mesocestoides spp. may develop through a two-host life-cycle only, using vertebrates as the intermediate host. Mesocestoides spp. have been recognized for their problematic taxonomic status and the lack of good morphological diagnostic features, with the validity of many species being questioned by numerous authors until the onset of genetic evaluations [11, 12]. In birds, the records of Mesocestoides spp. larvae are rare and are not limited to any family or order [8, 13-18]. However, occasionally the prevalence can be massive, such as in the Alectoris graeca populations examined in Greece [19]. The infections in birds may affect various tissues and organs. The body cavity is the most common infection site, where Mesocestoides larvae may cause disease termed peritoneal larval cestodiasis [13, 17, 20-23]. However, other sites may also become infected; these include the peritoneum [20], lungs [20], air sacs [13], intercostal muscles [15], liver [20, 22] and subcutaneous connective tissue [22]. The tetrathyridia could also be present in cutaneous cysts [17].

With regards to studies that employed DNA sequencing, several DNA loci were examined, including $12 S$ ribosomal deoxyribonucleic acid (rDNA) [10, 12, 24-30], cytochrome $c$ oxidase subunit 1 (cox 1$)[10,24,25,28$, 30-34], nicotinamide adenine dinucleotide dehydrogenase subunit 1 (nad1) [10, 28, 32, 34], $18 S$ rDNA [29, 32, 35-37], internal transcribed spacer 2 (ITS2) [12, 35] and $28 S$ rDNA [24, 32, 37, 38]. Sequences of Mesocestoides spp. from bird hosts were provided by Literák et al. [17], who sequenced $18 \mathrm{~S}$ rDNA of Mesocestoides sp. from thoracic cavity of a common starling, Sturnus vulgaris, from Czechia, as well as by Skirnisson et al. [24] reporting several loci of tetrathyridia from the rock ptarmigan, Lagopus muta, from Iceland. Despite the voluminous literature, phylogenetic analyses indisputably recognize only M. canislagopodis, M. litteratus, Mesocestoides lineatus (Goeze, 1782) and Mesocestoides corti Hoeppli, 1925/M. vogae Etges, 1991 [24], whereas the number of morphospecies varies between 12 [15] and 27 [39]. Moreover, the names $M$. corti and $M$. vogae were used for larval stages only and their taxonomic status remains uncertain [16].

In the present study, we aimed to investigate a massive and lethal infection of a song thrush, Turdus philomelos, by Mesocestoides sp. tetrathyridia. We performed combined morphological and phylogenetic analysis of the tetrathyridia and compared them with the materials obtained previously from other birds and mammals, identifying a match with a large number of isolates that were recently reported from domestic dogs and cats across Europe, the Middle East and North Africa.

\section{Results}

The examined T. philomelos had cysts of Mesocestoides sp. tetrathyridia across the whole body cavity, from the surface of lungs through liver, intestine and kidneys. The tetrathyridia were encysted individually or in small groups up to five individuals in a single cyst; in total, 104 tetrathyridia were present. The examined bird displayed signs of emaciation, the ovary was defunct and the eggs were up to $1 \mathrm{~mm}$ in diameter.

The morphologically examined tetrathyridia were 1641-4375 $\mu \mathrm{m}(2727 \pm 597 \mu \mathrm{m}, n=36)$ long and $1247-$ $1903 \mu \mathrm{m}(1592 \pm 122 \mu \mathrm{m}, n=36)$ wide. Their body was always longer than wide (Fig. $1 \mathrm{a}-\mathrm{c}$ ), with a length to width ratio of $1.03-2.67 \mu \mathrm{m}(1.71 \pm 0.31 \mu \mathrm{m}, n=36)$. Anterior end was invaginated. In $72 \%$ of individuals, scolex and neck were invaginated into body of metacestode, thus forming curved anterior canal (Fig. 1d) with variable length and width, 202-975 $\mu \mathrm{m}(546 \pm 154 \mu \mathrm{m}$, $n=26)$ long and $36-138 \mu \mathrm{m}(72 \pm 21 \mu \mathrm{m}, n=26)$ wide, with suckers often forming its bottom. Suckers were oval, with diameter 121-201 $\mu \mathrm{m}(165 \pm 14 \mu \mathrm{m}, n=34)$. Posterior end of body was invaginated, with pore of osmoregulatory system sometimes distinct in middle of invagination.

The molecular analyses revealed that the examined species was nested within other Mesocestoides spp. but was not identical with any Mesocestoides spp. previously identified at the species level and subjected to genetic analyses (Figs. 2, 3, Additional file 1: Figure S1, Additional file 2: Figure S2, Additional file 3: Figure S3, Additional file 4: Figure S4, Additional file 5: Figure S5, Additional file 6: Figure S6). The $12 S$ rDNA locus sequence divergences of $M$. lineatus, $M$. canislagopodis, $M$. corti/M. vogae and $M$. leptothylacus from the analyzed species were $10.7 \pm 2.3 \%, 12.8 \pm 2.9 \%, 4.3 \pm 1.1 \%$ and $12.7 \pm 2.6 \%$, respectively (Additional file 7: Table S1). $12 S$ rDNA was unavailable for $M$. litteratus; this species has a divergence of $15.0 \pm 2.6 \%$ in the $\operatorname{cox} 1$ locus, the sequence divergence of which reached $13.2-15.1 \%$ for other examined Mesocestoides spp. that were identified to species. The $18 \mathrm{~S}$ rDNA sequence of the previous genetically characterized isolate from birds, M. litteratus (GenBank: AY426258, [17]), was similar to the corresponding segment of nuclear rDNA (divergence only $0.2 \pm 0.2 \%$ ). However, $18 S \mathrm{rDNA}$ is not considered a hypervariable marker and other previously sequenced Mesocestoides spp. had just slightly higher divergence in this locus; these included M. corti (GenBank: AF286984 [38]) (with a divergence of $0.3 \pm 0.3 \%$ ) and M. litteratus (GenBank: DQ643000 [40]) (with a divergence of $1.0 \pm 0.4 \%$ ). The $12 S \mathrm{rDNA}$ of the analyzed species was also highly similar to those from larvae that were previously isolated from life-threatening 


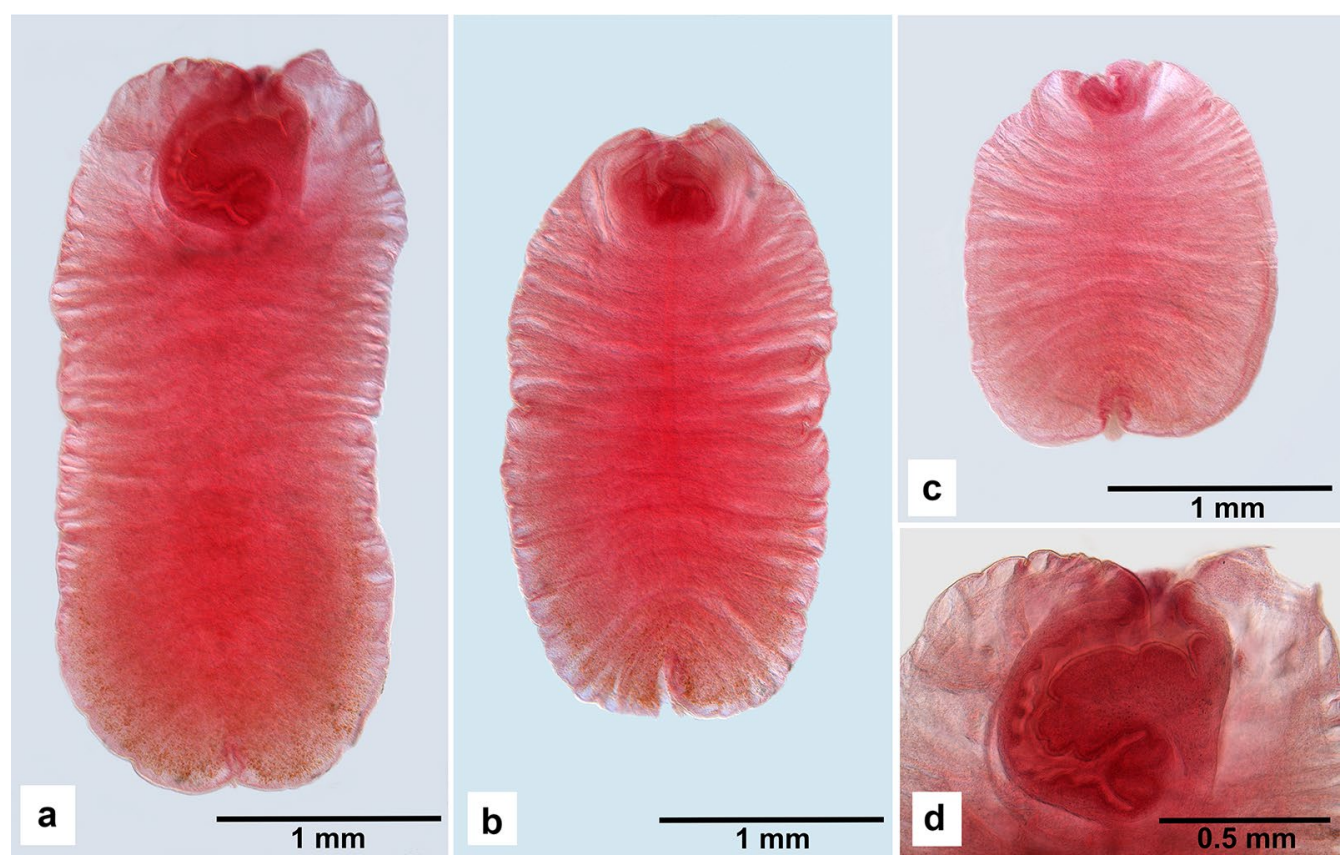

Fig. 1 Tetrathyridia of Mesocestoides sp. found in the body cavity of Turdus philomelos in Czechia (iron acetocarmine, dimethyl phthalate, Canada balsam). a-c Individuals demonstrating variations in size and shape of tetrathyridia. Note the invaginated scolex and neck (transformed into "anterior canal") as well as the presence of posterior invagination in all individuals. $\mathbf{d}$ Anterior end of the individual presented in $\mathbf{a}$. Note the curved "anterior canal" representing the scolex and the neck invaginated into the body of the metacestode. The apical part of the scolex bearing suckers is distinct at the bottom of the canal

infections of dogs in Turkey and identified as $M$. corti HM011122 [41], with a divergence of $0.7 \pm 0.5 \%$, and JN572111 [42], with a divergence of $0.7 \pm 0.9 \%$. However, these tetrathyridia clearly do not represent $M$. corti as other individuals, identified as $M$. corti by Kashiide et al. (GenBank: AB848990 [25]) and Nakao (GenBank: AB031363; unpubl.) formed a distinct clade (Fig. 2c). Moreover, other loci, for which $M$. corti was sequenced, did not agree with the identification of the present material as $M$. corti (Figs. 2, 3).

Importantly, besides the above-described associations, the analyzed species was highly similar to or identical with numerous Mesocestoides larvae that were not identified at the species level and that were recently sequenced from domestic dogs and cats. The loci with zero sequence divergence included the cox 1 locus of the larva from a domestic cat (GenBank: JQ740884 [33]) (Fig. 2a); $12 \mathrm{~S}$ rDNA locus of larvae from peritoneal cavities of domestic dogs (GenBank: MH992709, MH992714-MH992716; all unpublished) (Fig. 2c); and 18S-ITS1-5.8S-ITS2 locus of a larva from a domestic dog (GenBank: KP941433 [43]) (Fig. 3a, b). Recently, Varcasia et al. [34] reported a series of findings of larvae of this species from domestic dogs of south Italian, Sicilian and Tunisian origin. In addition to larval isolates, the isolate TUN01 ( $\operatorname{cox} 1$ sequence MH463506), which originated from an intestine of a domestic dog from Tunisia, represented the first and only record of an adult individual that is genetically matched to the individuals examined in the present study. However, the authors did not identify this isolate to species and labeled it instead as the cluster "M3" and did not reveal any details concerning its morphology.

The sequences with high similarity (but not complete identity) included $\operatorname{cox} 1$ and nad 1 sequences of another series of domestic dogs and cats from south Italy, Sardinia, Sicily and Tunisia [34]; the nad 1 sequence of an isolate from a domestic dog (Hirzmann et al. personal communication) (Fig. 2b); and the $12 \mathrm{~S}$ rDNA sequence of larvae from domestic dogs (GenBank: MH992719 (unpublished) and KP941431 [43]) (Fig. 2c). The Bayesian inference analysis corroborated all the above conclusions (Additional file 7: Figure S7, Additional file 8: Figure S8, Additional file 9: Figure S9, Additional file 10: Figure S10, Additional file 11: Figure S11, Additional file 12: Figure S12; the colors of the branches indicate Bayesian posterior probabilities).

Combined, the molecular analyses suggest that the presently examined isolate represented a distinct species of Mesocestoides that was previously repeatedly isolated as an unidentified larval and adult form from canine 


\section{a $\operatorname{cox} 1$}

MH463501 Mesocestoides sp. ex Canis lupus MH463506 Mesocestoides sp. ex Canis lupus MH463494 Mesocestoides sp. ex Canis lupus MH463492 Mesocestoides sp. ex Canis lupus

69 MK239658 Mesocestoides sp. ex Turdus philomelos

JQ740884 Mesocestoides sp. ex Felis silvestris

MH463505 Mesocestoides sp. ex Canis lupus

MH463511 Mesocestoides sp. ex Canis lupus

MH463507 Mesocestoides sp. ex Canis lupus

41 MH463497 Mesocestoides sp. ex Canis lupus

51 MH463498 Mesocestoides sp. ex Canis lupus

61 MH463499 Mesocestoides sp. ex Canis lupus

MH463500 Mesocestoides sp. ex Canis lupus

KY434329 Mesocestoides sp. ex Canis lupus

MH463495 Mesocestoides sp. ex Canis lupus

MH463503 Mesocestoides sp. ex Canis lupus

MH463503 Mesocestoides sp. ex Canis lupus
MH463493 Mesocestoides sp. ex Felis catus

68

99 MK095584 Mesocestoides vogae ex Canis lupus (rev. compl.)

KP941432 Mesocestoides sp. ex Canis lupus

MH463496 Mesocestoides sp. ex Canis lupus

MH463508 Mesocestoides sp. ex Canis lupus

62 MH463509 Mesocestoides sp. ex Canis lupus

MH463510 Mesocestoides sp. ex Canis lupus

KU821650 Mesocestoides sp. ex Felis silvestris

MH463504 Mesocestoides sp. ex Vulpes vulpes

MH463491 Mesocestoides sp. ex Felis catus

$100-$ MH463502 Mesocestoides sp. ex Felis catus

44 MF537039 Mesocestoides sp. ex Saguinus midas

MF537040 Mesocestoides sp. ex Saguinus midas

KT232150 Mesocestoides canislagopodis ex Canis lagopus

99 AB792715 Mesocestoides lineatus ex Vulpes vulpes

AB033413 Mesocestoides corti ex undisclosed host

100 AB848991 Mesocestoides vogae ex Canis lupus

JF268517 Mesocestoides litteratus ex Vulpes vulpes

MG323573 Mesocestoides sp. ex Scincella lateralis

KP161210 Echinococcus equinus (Taeniidae)

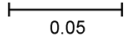

0.05

b $\operatorname{nad} 1$

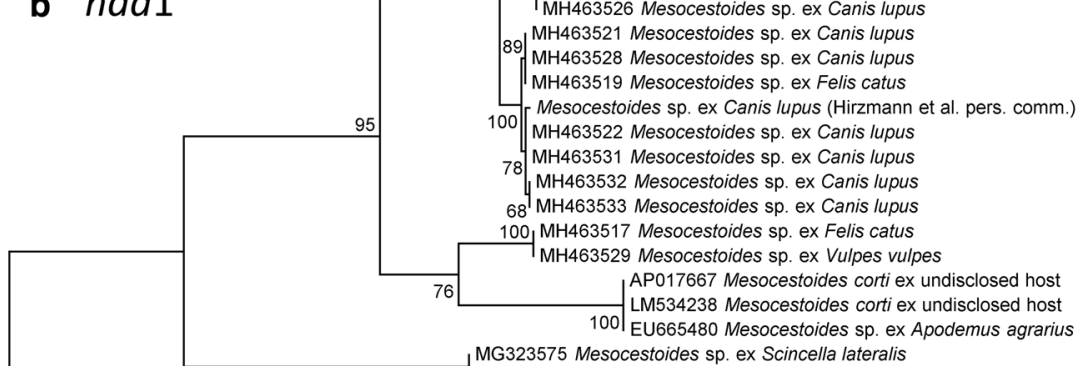

${ }_{100}^{\text {MG323575 Mesocestoides sp. ex Scincella lateralis }} \begin{aligned} & \text { MG323574 Mesocestoides sp. ex Scincella lateralis } \\ & \text { MG }\end{aligned}$

MH463527 Mesocestoides sp. ex Canis lupus

MH463530 Mesocestoides sp. ex Canis lupus

MH463520 Mesocestoides sp. ex Canis lupus

100 MK239659 Mesocestoides sp. ex Turdus philomelos

MH463518 Mesocestoides sp. ex Canis lupus

MH463523 Mesocestoides sp. ex Canis lupus

MH463524 Mesocestoides sp. ex Canis lupus

1001 MH463525 Mesocestoides sp. ex Canis lupus

MH463526 Mesocestoides Sp. ex Canis lupus

MH463528 Mesocestoides sp. ex Canis lupus

MH463522 Mesocestoides sp ex Canis lupus

MH463533 Mesocestoides sp. ex Canis lupus

sed host

LM534238 Mesocestoides corti ex undisclosed host

KX782315 Hymenolepis folkertsi (Hymenolepididae)

0.05

MK239660 Mesocestoides sp. ex Turdus philomelos

16 MH992716 Mesocestoides sp. ex Canis lupus

MH992715 Mesocestoides sp. ex Canis lupus

MH992715 Mesocestoides sp. ex Canis lupus

MH992709 Mesocestoides sp. ex Canis lupus
MH992714 Mesocestoides sp. ex Canis lupus

c $12 S \mathrm{rDNA}$

99 MH992719 Mesocestoides sp. ex Canis lupus

HM011122 Mesocestoides corti (vogae) ex Canis lupus

JN572111 Mesocestoides corti (vogae) ex Canis lupus

KP941431 Mesocestoides sp. ex Canis lupus

KT232152 Mesocestoides canislagopodis ex Vulpes lagopus

AB031363 Mesocestoides corti (vogae) ex undisclosed host

98 AB848990 Mesocestoides corti (vogae) ex Canis lupus

L49451 Mesocestoides leptothylacus ex Vulpes vulpes

MH992713 Mesocestoides sp. ex Canis lupus

85 EF567417 Mesocestoides lineatus ex Canis lupus



Fig. 2 Maximum likelihood analyses of the sequences of mitochondrial DNA loci of Mesocestoides spp.: a cox1; b nad1; and c 12S rDNA. The scale-bars indicate the number of substitutions per nucleotide site 


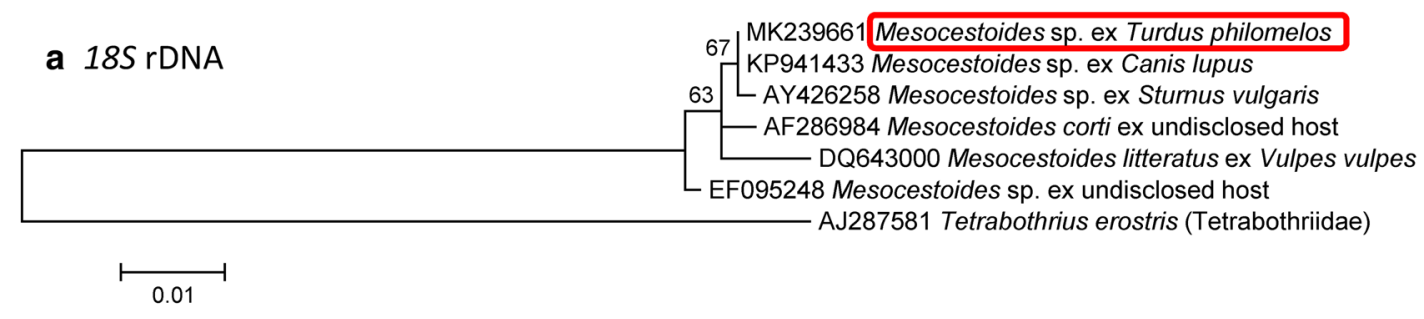

b $18 S-$ ITS1-5.8S-ITS2
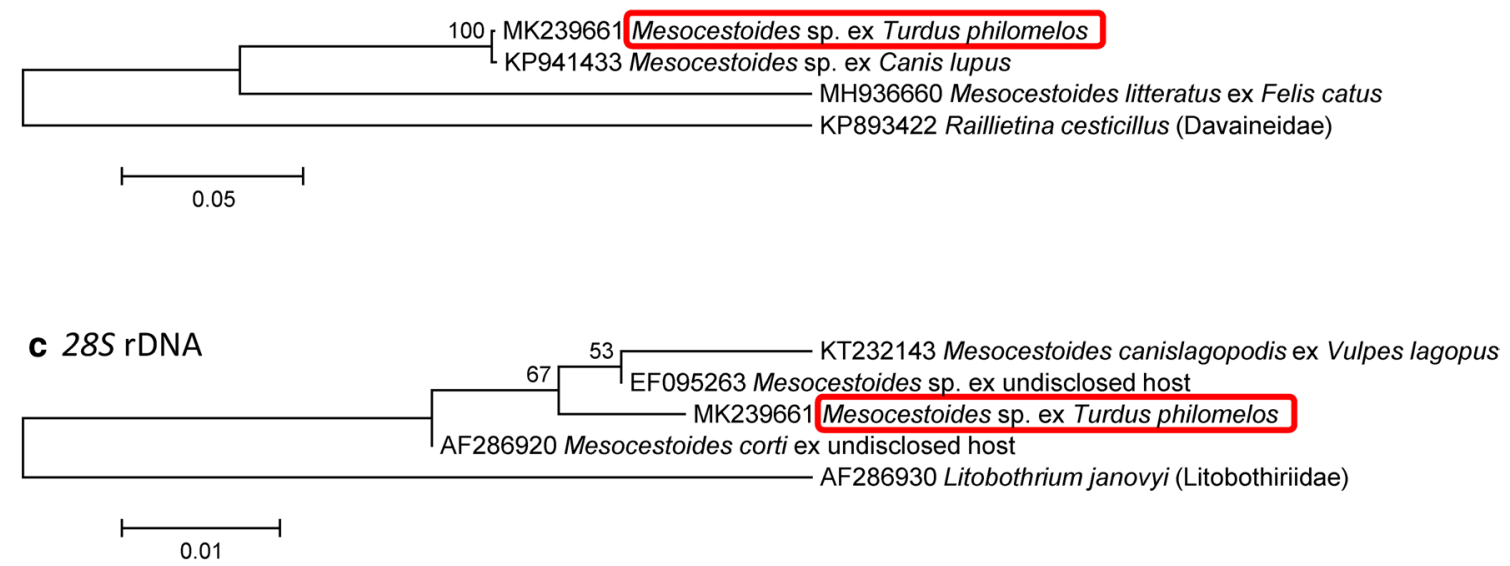

Fig. 3 Maximum likelihood analyses of the sequences of nuclear ribosomal DNA loci of Mesocestoides spp.: a 18S rDNA; b 3' part of 18S-ITS15.8S-ITS2; and c 285 rDNA. The bars indicate the number of substitutions per nucleotide site

and feline body cavities, with numerous DNA sequences available, but with no match to sequences of adult cestodes identified at the species level.

\section{Discussion}

The molecular analyses performed based on the isolate reported in the present study and previously reported isolates from body cavities of domestic dogs and cats suggest the presence of larvae of a species which does not match with sequences of any of the species identified on the basis of adult individuals. Before concluding that this is a new species, we should keep in mind that two further European species of Mesocestoides, Mesocestoides perlatus (Goeze, 1782) that parasitizes birds of prey and Mesocestoides melesi Yanchev \& Petrov, 1985 that parasitizes badgers $[15,40]$ have not been sequenced until now.

The morphology of the above-described metacestodes isolated from $T$. philomelos in Czechia is in agreement with previous descriptions of tetrathyridia of Mesocestoides [13-15, 17, 20, 21, 44-46]. The identification of tetrathyridia to the species level based on their morphology is, however, nearly impossible at the present stage $[18,29,44]$. For a more detailed morphological comparison of tetrathyridia, see Literák et al. [17]. However, we believe that morphological documentation of the records is important in view of further comparisons, when more numerous molecular data associated with morphological descriptions have been accumulated.

The isolates from mammals that were genetically similar or identical are described in detail below. Yildiz \& Tong [41] reported acephalic metacestodes free on the omental surfaces covering spleen, liver and small intestine of an examined dog, which manifested leukocytosis and an increased hematocrit, but did not provide any morphological details. Their diagnosis of the metacestodes as $M$. corti is here rejected based on the phylogenetic analyses performed in the present study (Fig. 2c). Aypak et al. [42] reported abundant acephalic metacestodes alongside numerous empty calcified cysts of a diameter less than $6 \mathrm{~mm}$ that were aggregated into multiple free aggregates of $15 \mathrm{~mm}$ in diameter in the peritoneum of an examined dog. The dog manifested normocytic normochromic anemia with low red blood cell count and low hemoglobin, and neutrophilic leukocytosis. Diffuse ascites were present and the peritoneal cysts caused the presence of diffusely scintillating abdominal hyperechoic foci as detected by an X-ray examination. Fibroproliferative peritonitis, chronic gastritis with stomach ulcer and perforation, mycotic pyogranulomatous inflammation of spleen, proximal tubular epithelial degeneration and 
interstitial plasma cell infiltration of the kidneys and passive hyperemia and fibrosis of the liver were described. Consistent with the above-described case, the larvae did not have distinct scolex and sucker structures that would otherwise be characteristic for the tetrathyridia; the larvae were described as covered by a thick eosinophilic integument. The authors believed that they observed the asexual reproduction of acephalic metacestodes by budding, which is consistent with Galán-Puchades et al. [47], who identified three patterns of asexual reproduction in metacestodes of the genus Mesocestoides: longitudinal fission, budding and development of daughter metacestodes in a mother larva. The issue of asexual reproduction of Mesocestoides larvae remains controversial, with Specht \& Voge [48], Etges [49] and other authors arguing for its presence in laboratory animals. Conn [50] criticized the multiple records of asexually reproducing Mesocestoides larvae as doubtful, believing this phenomenon to be well-documented only for a single population of $M$. corti from California studied by Specht \& Voge [48]. In the present study, we did not observe any sign of asexual reproduction among the morphologically examined tetrathyridia. Recently, Varcasia et al. [34] reported numerous genetically matched cases from domestic dogs and cats from Italy and Tunisia but did not provide any details on the infection symptoms except of the localization of infection.

Concerning other genetically matched cases of infections, Jabbar et al. [33] reported an infected cat, which displayed severe pan-leucopenia, increased liver enzyme concentrations in blood, namely increased $\gamma$-glutamyl transferase and aspartate aminotransferase, and mild azotemia. The animal displayed a right ventricular, hypertrophic cardiomyopathy and generalized lung damage, with areas of parenchymal collapse, carnification and discoloration, consistent with chronic respiratory disease. Macroscopic examination of the abdominal cavity and visceral organs did not reveal any parasites but a thoracotomy, by midline sternotomy, detected nine acephalic metacestodes, being 1-5 $\mathrm{cm}$ in length in the thoracic cavity. Similarly to above cases, the authors did not provide any additional morphological details. Finally, Häußler et al. [43] reported a dog with a hyperechoic mass in the middle abdomen detected by sonography and X-ray and with a middle grade systemic inflammatory reaction, mild anemia and low albumin and high globulin serum concentrations. Consistently with the three above studies, the larvae were described as lacking scolex and sucker structures, thus they have to be considered acephalic metacestodes (despite the authors calling them tetrathyridia). These larvae were $0.5-1.5 \mathrm{~cm}$ in length, with no further morphological details being provided.
Combined, four of the five previous studies that identified the examined species in mammals reported the agent as the acephalic metacestode stage. It remains unclear, however, if these were metacestodes in the process of development, at the stage before differentiation of the scolex and neck, or if these are developed individuals with an invaginated scolex and neck, which can be visualized by application of staining and/or clearing techniques (Fig. 1). Tetrathyridia, and even an adult individual of the genetically matched individuals, were recently reported from Italy and Tunisia [34]. The study characterized the species using the mitochondrial DNA; however, it did not reveal any details concerning the morphology of tetrathyridia and even of the examined adult individual.

The genetic correspondence of the tetrathyridia found by us with adult worms isolated from the intestines of a Tunisian dog is not surprising in view of the migration of the avian host. The song thrush (Turdus philomelos) is a breeding bird in Central Europe. The bird examined by us in the very early spring was obviously found soon after its spring migration. Based on recoveries, more than half of birds ringed in their nesting areas in central Europe were recognized as wintering in Maghreb [51].

\section{Conclusions}

In the present study, we describe a rare and likely lethal case of an infection of a songbird by Mesocestoides sp. tetrathyridia. Phylogenetic analyses revealed that the examined isolates are genetically identical or similar to isolates from domestic dogs and cats reported across the Western Palearctic. Four of the five previous studies described infection by the acephalic metacestode stage only; the fifth study, which reported tetrathyridia and an adult individual, did not reveal any morphological details. The possible identity with species previously reported to infect birds remains uncertain. The newly provided DNA sequences should allow the assignment to species in future when adults of the genus Mesocestoides are more thoroughly sequenced.

\section{Methods}

Necropsy of a free-ranging female of Turdus philomelos, found dead in the vicinity of Záhlinice (eastern Czechia, $49^{\circ} 16^{\prime} \mathrm{N}, 17^{\circ} 28^{\prime} \mathrm{E}$ ) on April 9, 2018, revealed the presence of a large number of tetrathyridia in cysts that were localized across the whole body cavity. For the identification of parasites, we collected all the tetrathyridia and fixed them in $96 \%$ ethanol for further analyses. For the comparative morphological analyses, we stained a set of 36 individuals with iron acetocarmine as described by Georgiev et al. [52], dehydrated in an ascending ethanol series (70, 80, 90, 96 and 100\%), cleared in dimethyl phthalate 
and mounted in Canada balsam. Their metrical data are presented as the range followed (in parentheses) by the mean \pm standard deviation (SD) and the number of measurements taken $(n)$. All measurements are provided in $\mu \mathrm{m}$. Voucher specimens are deposited in the Invertebrate Collection of the Natural History Museum of Geneva (four slides, 16 specimens), acquisition no. MHNGPLAT-121610, and in the Helminthological Collection of the Institute of Biodiversity and Ecosystem Research, Bulgarian Academy of Sciences, Sofia (five slides, 20 specimens), acquisition no. IBER-BAS-C-0159.1.6-1.10.

For molecular examination, we extracted, amplified and sequenced the DNA using the primers that targeted the nuclear ribosomal DNA (18S-ITS1-5.8S-ITS2 and $28 S$ rDNA) and three mitochondrial loci (cox1, nad 1 and $12 S \mathrm{rDNA}$ ) as described [53]. The primers used are specified in Additional file 14: Table S2. The resulting consensus sequences were submitted to the GenBank database under the accession numbers MK239658 (cox1), MK239659 (nad1), MK239660 (12S rDNA) and MK239661 (nuclear ribosomal DNA).

We aligned the newly generated sequences, sequences of Mesocestoides spp. obtained from GenBank as of November 30, 2018, and sequences of corresponding outgroups using ClustalW in MEGA5 (gap opening penalty 7 and gap extension penalty 2 for both pairwise and multiple alignments, DNA weight matrix IUB, and transition weight 0.1 ). We manually checked and corrected the inconsistencies. We trimmed each alignment to the length of the shortest included sequence. Because the Mesocestoides spp. sequences that were available in GenBank did not cover the whole range of the nuclear rDNA sequenced in the present study, we performed three separate alignments of parts of the nuclear rDNA that corresponded to partial $18 S$ rDNA, the ITS1-5.8SITS2 segment and the $28 S$ rDNA. The trimmed cox 1 locus (partial cox 1 coding sequence) corresponded to nt. 13-369 (357 bp) of M. lineatus AB792715 (Additional file 1: Figure S1). The trimmed nad 1 locus (partial nad1 coding sequence) corresponded to nt. 5309-5720 (412 bp) of $M$. corti AP017667 (Additional file 2: Figure S2). The trimmed $12 S$ rDNA locus (partial $12 S$ ribosomal RNA coding sequence) corresponded to nt. 7-299 (293 bp) of M. corti AB031363 (Additional file 3: Figure S3). The trimmed $18 S$ rDNA locus [partial small subunit rRNA coding sequence] corresponded to nt. 370-986 (617 bp) of Mesocestoides sp. AY426258 (Additional file 4: Figure S4). The trimmed 18S-ITS1-5.8S-ITS2 locus (partial $18 S$ rRNA coding sequence, full-length ITS1, fulllength 5.8S ribosomal RNA coding sequence and partial ITS2 sequences) corresponded to nt. 1459-2689 (1231 bp) of Mesocestoides sp. KP941433 (Additional file 5: Figure S5). The trimmed $28 \mathrm{~S}$ rDNA locus [partial large subunit ( $L S U$ ) rRNA coding sequence] corresponded to nt. 49-301 (253 bp) of M. canislagopodis KT232143 (Additional file 6: Figure S6). We analyzed the maximum likelihood fits of the 24 nucleotide substitution models in MEGA5. The best-fitting models according to the Bayesian information criterion were GTR + I (for $\operatorname{cox} 1$ ), $\mathrm{HKY}+\mathrm{I}$ (for nad1), HKY + G (for $12 S \mathrm{rDNA}$ ), K2 (for $18 S$ rDNA), HKY (for the ITS1-5.8S-ITS2 locus and for the $28 \mathrm{~S}$ rDNA locus, respectively). We used bootstrapping at 1000 replicates and the nearest-neighbor-interchange as the maximum likelihood heuristic method of choice to infer the trees and used best-fit models for the maximum likelihood phylogenetic analyses. To analyze the evolutionary divergence of the analyzed species from those that were sequenced previously, we estimated the pairwise distances expressed as a number of base differences per site obtained by averaging over all sequence pairs between groups. The data are shown as the means resulting from the application of the bootstrap procedure with 1000 replicates.

To corroborate the results of maximum likelihood analyses, we performed Bayesian inference analysis in MrBayes v. 3.2.5 using the mixed model of nucleotide substitution and including four Monte Carlo Markov chains for 10,000,000 generations. We discarded the first $25 \%$ of the samples as burn-in and used the remaining data to generate a $50 \%$ majority-consensus tree with the indicated posterior probabilities of branches. The obtained summary statistics are provided in Additional file 15: Table S3.

\section{Additional files}

Additional file 1: Figure S1. Alignment of the trimmed cox 1 locus (partial cox1 coding sequence).

Additional file 2: Figure S2. Alignment of the trimmed nad1 locus (partial nad1 coding sequence).

Additional file 3: Figure S3. Alignment of the trimmed 12S rDNA locus (partial 125 ribosomal RNA coding sequence).

Additional file 4: Figure S4. Alignment of the trimmed 18S rDNA locus (partial SSU rRNA coding sequence).

Additional file 5: Figure S5. Alignment of the trimmed 18S-ITS1-5.8SITS2 locus (partial 18S rRNA coding sequence, full-length ITS1, full-length 5.85 ribosomal RNA coding sequence and partial ITS2 sequences).

Additional file 5: Figure S6. Alignment of the trimmed 285 rDNA locus (partial LSU rRNA coding sequence).

Additional file 7: Table S1. The 12S rDNA locus sequence divergences of M. lineatus, M. canislagopodis, M. corti/vogae, M. leptothylacus and M. litteratus from the presently analyzed species. Data are shown as mean \% of divergence (below the diagonal), with S.E. shown above the diagonal.

Additional file 8: Figure S7. Phylogenetic tree based on Bayesian inference of the cox 1 of Mesocestoides.

Additional file 9: Figure S8. Phylogenetic tree based on Bayesian inference of the nad 1 of Mesocestoides. 
Additional file 10: Figure S9. Phylogenetic tree based on Bayesian inference of the 12S rDNA of Mesocestoides.

Additional file 11: Figure S10. Phylogenetic tree based on Bayesian inference of the 185 rDNA of Mesocestoides.

Additional file 12: Figure S11. Phylogenetic tree based on Bayesian inference of the ITS1-5.8S rDNA-ITS2 of Mesocestoides.

Additional file 13: Figure S12. Phylogenetic tree based on Bayesian inference of the 28S rDNA of Mesocestoides.

Additional file 14: Table S2. List of primers that were used to amplify the analyzed DNA loci.

Additional file 15: Table S3. Summary statistics for Bayesian inference analyses performed. The data are provided for partitions with frequency $\geq 0.10$ in at least one run.

\section{Abbreviations}

Cox1: cytochrome c oxidase, subunit 1; ITS2: internal transcribed spacer 2; LSU: large subunit; $n$ : number of measurements taken; nad1: nicotinamide adenine dinucleotide dehydrogenase, subunit 1; rDNA: ribosomal deoxyribonucleic acid; SD: standard deviation; SSU: small subunit.

\section{Acknowledgements}

We thank Jörg Hirzmann and Thomas Häußler (Justus-Liebig-Universität Gießen, Germany) for sharing the nad 1 sequence of their Mesocestoides sp. isolate and for the discussion of our initial findings, Gergana Vasileva (Bulgarian Academy of Sciences, Bulgaria) for her kind assistance in preparing Fig. 1 and Miroslav Těšínský (Charles University, Czechia) for the assistance with the molecular part of the study.

\section{Authors' contributions}

IL and PH conceived the study, PH performed the molecular analyses, BBG performed the morphological and morphometrical analyses, JS performed the parasitological examination of the host bird, PH and BBG wrote the manuscript. All authors critically revised previous manuscript versions. All authors read and approved the final manuscript.

\section{Funding}

Not applicable. The authors received no specific funding for this work.

\section{Availability of data and materials}

Voucher specimens have been deposited in the Invertebrate Collection of the Natural History Museum of Geneva (4 slides, 16 specimens), acquisition no. MHNG-PLAT-121610, and in the Helminthological Collection of the Institute of Biodiversity and Ecosystem Research, Bulgarian Academy of Sciences, Sofia (5 slides, 20 specimens), acquisition no. IBER-BAS-C-0159.1.6-1.10. The newly generated consensus DNA sequences were submitted to the GenBank database under the accession numbers MK239658 (cox1), MK239659 (nad1), MK239660 (12S rDNA) and MK239661 (nuclear ribosomal DNA).

\section{Ethics approval and consent to participate}

Not applicable.

\section{Consent for publication}

Not applicable.

\section{Competing interests}

The authors declare that they have no competing interests.

\section{Author details}

${ }^{1}$ Third Faculty of Medicine, Charles University, Prague, Czechia. ${ }^{2}$ Institute of Biodiversity and Ecosystem Research, Bulgarian Academy of Sciences, Sofia, Bulgaria. ${ }^{3}$ Comenius Museum, Moravian Ornithological Station, Přerov, Czechia. ${ }^{4}$ Department of Biology and Wild life Diseases, Faculty of Veterinary Hygiene and Ecology, University of Veterinary and Pharmaceutical Sciences Brno, Brno, Czechia.

Received: 18 December 2018 Accepted: 3 May 2019

Published online: 14 May 2019

\section{References}

1. Spasskaja LP. Cestodes of birds of Tuva. V. Species from Galliformes and some rapacious birds. Acta vet Acad Sci Hung. 1962;12:423-44 (In Russian).

2. Loos-Frank B. Cestodes of the genus Mesocestoides (Mesocestoidae) from carnivores in Israel. Isr J Zool. 1990;37:3-13.

3. Sanmartín ML, Ivarez FA, Barreiro G, Leiro J. Helminth fauna of Falconiform and Strigiform birds of prey in Galicia, Northwest Spain. Parasitol Res. 2004;92:255-63.

4. Komorová P, Sitko J, Špakulová M, Hurníková Z, Sałamantin R, Chovancová G. New data on helminth fauna of birds of prey (Falconiformes, Accipitriformes, Strigiformes) in the Slovak Republic. Helminthologia. 2017;54:314-21.

5. Montalbano Di Filippo M, Meoli R, Cavaller S, Eleni C, De Liberato C, Berrilli F. Molecular identification of Mesocestoides sp. metacestodes in a captive gold-handed tamarin (Saguinus midas). Infect Genet Evol. 2018;65:399-405.

6. Chandler AC. First record of a case of human infection with tapeworms of the genus Mesocestoides. Am J Trop Med Hyg. 1942;S1-22:493-7.

7. Fuentes MV, Galan-Puchades MT, Malone JB. A new case report of human Mesocestoides infection in the United States. Am J Trop Med Hyg. 2003;68:566-7.

8. Prudhoe S, Bray RA. Platyhelminth parasites of the Amphibia. London \& Oxford: British Museum \& Oxford University Press; 1982.

9. Tenora F. Notes to Mesocestoides Vaillant, 1863 (Cestoda) and findings of Mesocestoides sp. parasitizing Canis familiaris (Carnivora) in the Czech Republic. Acta Univ Agric Silvicult Mendel Brun. 2004;52:25-33.

10. McAllister CT, Tkach VV, Conn DB. Morphological and molecular characterization of post-larval pre-tetrathyridia of Mesocestoides sp. (Cestoda: Cyclophyllidea) from ground skink, Scincella lateralis (Sauria: Scincidae), from southeastern Oklahoma. J Parasitol. 2018;104:246-53.

11. Loos-Frank B. Mesocestoides leptothylacus n. sp. und das nomenklatorische Problem in der Gattung Mesocestoides Vaillant, 1863 (Cestoda, Mesocestoididae). Tropenmed Parasitol. 1980;1980(31):2-14.

12. Padgett KA, Nandler SA, Munson L, Sacks B, Boyce WM. Systematics of Mesocestoides (Cestoda: Mesocestoididae): evaluation of molecular and morphological variation among isolates. J Parasitol. 2005;91:1435-43.

13. Joyeux C, Baer J-G. Faune de France. 30. Cestodes. Paris: P. Lechevalier; 1936.

14. Gvozdev EV. Parasitic worms of galliform birds of Kazakhstan. Alma-Ata: Izdatel'stvo Akademii Nauk Kazakhskoy SSR; 1958 (In Russian).

15. Chertkova AN, Kosupko GA. Suborder Mesocestoidata Skrjabin, 1940. In: Ryzhikov KM, editor. Principles of cestodology, vol. 9. Moscow: Nauka; 1978. p. 118-229 (In Russian).

16. Rausch RL. Family mesocestoididae fuhrmann, 1907. In: Khalil LF, Jones A, Bray RA, editors. Keys to the cestode parasites of vertebrates. Wallingford: CAB International; 1994. p. 309-14.

17. Literák I, Olson PD, Georgiev BB, Špakulová M. First record of metacestodes of Mesocestoides sp. in the common starling (Sturnus vulgaris) in Europe, with an 185 rDNA characterisation of the isolate. Fol Parasitol. 2004;51:45-9.

18. Skirnisson K, Sigurðardóttir ÓG, Nielsen ÓK. Morphological characteristics of Mesocestoides canislagopodis (Krabbe, 1865) tetrathyridia found in rock ptarmigan (Lagopus muta) in Iceland. Parasitol Res. 2016;115:3099-106.

19. Manios N, Papazahariadou M, Frydas S, Papageorgiiu N, Tsachalidis E, Georgopoulou J. Tetrathyridium as a mortality factor of rock partridge (Alectoris graeca graeca) in Central Greece. Z Jagdwiss. 2002;48:378-82.

20. Chertkova AN, Petrov AM. Helminth parasites of domesticated galliform birds and the diseases caused by them, vol. 1. Moscow: All-Union Institute of Helminthology; 1959 (In Russian).

21. Oshmarin GP. Parasitic worms of mammals and birds in Primor'e region. Moscow: Izdatel'stvo Akademii Nauk SSSR; 1963 (In Russian).

22. Borgarenko LF. Helminth parasites of birds in Tadzhikistan. Book 1. Cestodes. Dushanbe: Donish; 1981 (In Russian)

23. Millán J, Gortazar G, Casanova JC. First occurrence of Mesocestoides sp. in a bird, the red-legged partridge, Alectoris rufa, in Spain. Parasitol Res. 2003;90:80-1.

24. Skirnisson K, Jouet D, Ferté H, Nielsen OK. Mesocestoides canislagopodis (Rudolphi, 1810) (Krabbe 1865) in mammals and birds and its molecular discrimination within the Mesocestoides species complex. Parasitol Res. 2016;115:2597-607. 
25. Kashiide T, Matsumoto J, Yamaya Y, Uwasawa A, Miyoshi A, Yamada K, et al. Case report: first confirmed case of canine peritoneal larval cestodiasis caused by Mesocestoides vogae (syn. M. corti) in Japan. Vet Parasitol. 2014;201:154-7

26. von Nickisch-Rosenegk M, Lucius R, Loos-Frank B. Contributions to the phylogeny of the Cyclophyllidea (Cestoda) inferred from mitochondrial 12S rDNA. J Mol Evol. 1999;48:586-96.

27. Wirtherle N, Wiemann A, Ottenjann M, Linzmann H, Grinten E, Kohn B, et al. First case of canine peritoneal larval cestodosis caused by Mesocestoides lineatus in Germany. Parasitol Int. 2007;56:317-20.

28. Hrčková G, Miterpáková M, O'Connor A, Šnábel V, Olson PD. Molecular and morphological circumscription of Mesocestoides tapeworms from red foxes (Vulpes vulpes) in central Europe. Parasitology. 2011;138:638-47.

29. Zaleśny G, Hildebrand J. Molecular identification of Mesocestoides spp. from intermediate hosts (rodents) in central Europe (Poland). Parasitol Res. 2012;110:1055-61.

30. Yasur-Landau D, Salant H, Levin-Gichon G, Botero-Anug AM, Zafrany A, Mazuz ML, et al. Urinary incontinence associated with Mesocestoides vogae infection in a dog. Parasitol Res. 2019;118:1039-44.

31. Nakao M, Sako Y, Yokoyama N. Mitochondrial genetic code in cestodes. Mol Biochem Parasitol. 2000;111:415-24.

32. Littlewood DTJ, Waeschenbach A, Nikolov PN. In search of mitochondrial markers for resolving the phylogeny of cyclophyllidean tapeworms (Platyhelminthes, Cestoda) — a test study with Davaineidae. Acta Parasitol. 2008;53:133-44

33. Jabbar A, Papini R, Ferrini N, Gasser RB. Use of a molecular approach for the definitive diagnosis of proliferative larval mesocestoidiasis in a cat. Infect Genet Evol. 2012;12:1377-80.

34. Varcasia A, Sanna D, Casu M, Lahmar S, Dessì G, Pipia AP, et al. Species delimitation based on mtDNA genes suggests the occurrence of new species of Mesocestoides in the Mediterranean region. Parasit Vectors. 2018;11:619.

35. Crosbie PR, Nandler SA, Platzer EG, Kerner C, Mariaux J, Boyce WM. Molecular systematics of Mesocestoides spp. (Cestoda: Mesocestoididae) from domestic dogs (Canis familiaris) and coyotes (Canis latrans). J Parasitol. 2000;86:350-7.

36. Literák I, Tenora F, Letková V, Goldová M, Torres J, Olson PD. Mesocestoides litteratus (Batsch, 1786) (Cestoda: Cyclophyllidea: Mesocestoididae) from the red fox: morphological and 185 rDNA characterization of European isolates. Helminthologia. 2006;43:191-5.

37. Waeschenbach A, Webster BL, Bray RA, Littlewood DTJ. Added resolution among ordinal level relationships of tapeworms (Platyhelminthes: (estoda) with complete small and large subunit nuclear ribosomal RNA genes. Mol Phylogen Evol. 2007;45:311-25.

38. Olson PD, Littlewood DTJ, Bray RA, Mariaux J. Interrelationships and evolution of the tapeworms (Platyhelminthes: Cestoda). Mol Phylogen Evol. 2001;19:443-67.
39. Yamaguti S. Systema helminthum: the cestodes of vertebrates. New York: Interscience Publishers; 1959.

40. Yanchev Y. Morphology, taxonomy and distribution of the species of genus Mesocestoides Vaillant, 1863 in Bulgaria. Khelmintologiya. 1986;21:45-65 (In Bulgarian).

41. Yildiz K, Tong S. Canine peritoneal larval cestodosis in a dog. Tierärztl Prax. 2011;39:448-50.

42. Aypak S, Aysul N, Ural K, Biringioglu S, Atasoy A, Derincegöz O, et al. A case of diffuse peritoneal larval Mesocestoides corti (syn. M. vogae) cestodiasis in a dog in Turkey. Kafkas Univ Vet Fak. 2012;18:885-8.

43. Häußler TC, Peppler C, Schmitz S, Bauer C, Hirzmann J, Kramer M. Kanine peritoneale larvale Zestodose durch Mesocestoides spp. Tierärztl Prax K: Kleintiere/Heimtiere. 2016;44:123-8.

44. Kubečka BW, Traub NJ, Tkach W, Shirley TR, Rollins D, Fedynich A. Mesocestoides sp. in wild northern bobwhite (Colinus virginianus) and scaled quail (Callipepla squamata). J Wildl Dis. 2018;54:612-6.

45. Neveu-Lemaire M. Traité d'helminthologie médicale et vétérinaire. Paris: Vigot Frères; 1936.

46. Movsesyan SO. Cestodes of birds in transcaucasia. Yerevan: Izdatel'stvo Akademii Nauk Armyanskoi SSR; 1987 (In Russian).

47. Galán-Puchades MT, Fuentes MV, Conn DB. A new type of endogenous asexual proliferation in cyclophyllidean metacestodes. Acta Parasitol. 2002;47:288-93.

48. Specht $D$, Voge M. Asexual multiplication of Mesocestoides tetrathyridia in laboratory animals. J Parasitol. 1965;51:268-72.

49. Etges FJ. The proliferative tetrahydrium of Mesocestoides vogae sp. $\mathrm{n}$. (Cestoda). J Helminthol Soc Wash. 1991;58:181-5.

50. Conn DB. The rarity of asexual reproduction among Mesocestoides tetrathyridia (Cestoda). J Parasitol. 1990;76:453-5.

51. Milwright RDP. Post-breeding dispersal, breeding site fidelity and migration/wintering areas of migratory populations of Song Thrush Turdus philomelos in the Western Palearctic. Ringing Migr. 2006;23:21-32.

52. Georgiev B, Biserkov V, Genov T. In toto staining method for cestodes with iron acetocarmine. Helminthologia. 1986;23:279-81.

53. Heneberg P, Sitko J, Těšínský M, Rząd I, Bizos J. Central European Strigeidae Railliet, 1919 (Trematoda: Strigeidida): molecular and comparative morphological analysis suggests the reclassification of Parastrigea robusta Szidat, 1928 into Strigea Abildgaard, 1790. Parasitol Int. 2018;67:688-701.

\section{Publisher's Note}

Springer Nature remains neutral with regard to jurisdictional claims in published maps and institutional affiliations.
Ready to submit your research? Choose BMC and benefit from:

- fast, convenient online submission

- thorough peer review by experienced researchers in your field

- rapid publication on acceptance

- support for research data, including large and complex data types

- gold Open Access which fosters wider collaboration and increased citations

- maximum visibility for your research: over $100 \mathrm{M}$ website views per year

At BMC, research is always in progress.

Learn more biomedcentral.com/submissions 